IgG autoantibody levels $(P<0.05)$ towards another antigen, dual specificity mitogen-activated protein kinase kinase 6 (MAP2K6), were also observed in ACPA-seronegative subjects compared to ACPA-seropositive and controls. In contrast, we found significantly higher IgG autoantibody levels $(P<0.05)$ in ACPA-seropositive individuals compared to ACPA-seronegative and controls towards two antigens, anosmin-1 (ANOS-1) and muscle related coiled-coil protein (MURC). ANOS-1 shows also significantly higher IgG reactivity frequency in ACPA-seropositive individuals compared to ACPA-seronegative and controls $(22 \%, 9 \%$ and $6 \%$ respectively; $P<0.05)$. Interestingly, three out of the four antigens discovered to be associated with the ACPA status in early RA are highly expressed in lungs and heart, two of the main extraarticular sites affected in RA. No significant differences were observed at IgA levels for any of the antigens analyzed.

Table 1. Scheme of the different phases of the study, the features within each phase and the results. The reactivity to four antigens allows to distinguish ACPA-seronegative (ACPA-), ACPA seropositive (ACPA+) and controls.

\begin{tabular}{|c|c|c|c|}
\hline Phases & $\begin{array}{l}\text { Untargeted } \\
\text { discovery }\end{array}$ & $\begin{array}{l}\text { Targeted } \\
\text { discovery }\end{array}$ & $\begin{array}{l}\text { Targeted } \\
\text { validation }\end{array}$ \\
\hline Number of samples & $\begin{array}{l}80 \text { ACPA- } \\
80 \text { ACPA+ }\end{array}$ & $\begin{array}{l}80 \text { ACPA- } \\
80 \text { ACPA+ }\end{array}$ & $\begin{array}{l}358 \text { ACPA-372 Controls } \\
386 \text { ACPA+ }\end{array}$ \\
\hline $\begin{array}{l}\text { Antigen array } \\
\text { platform }\end{array}$ & $\begin{array}{l}\text { Planar } \\
\text { arrays }\end{array}$ & $\begin{array}{l}\text { Suspension } \\
\text { bead array } 1\end{array}$ & $\begin{array}{l}\text { Suspension } \\
\text { bead array } 2\end{array}$ \\
\hline Number of antigens & 2660 & 62 & 27 \\
\hline $\begin{array}{l}\text { Number of candidate } \\
\text { biomarkers }\end{array}$ & 62 & 27 & $\begin{array}{l}4 \text { (TSPYL4,MAP2K6, } \\
\text { ANOS1,MURC) }\end{array}$ \\
\hline
\end{tabular}

Conclusion: Upon further validation in other early RA sample cohorts, our data suggest the measurement of these four autoantibodies may be useful for the early diagnosis of ACPA-seronegative RA and give insight into the pathogenesis of the different RA subsets.

Characters from table content including title and footnotes:

Disclosure of Interests: None declared

DOI: 10.1136/annrheumdis-2021-eular.2514

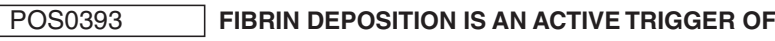 CARTILAGE DEGENERATION IN RHEUMATOID ARTHRITIS}

T. Hügle ${ }^{1}$, S. Nasi ${ }^{1}$, D. Ehirchiou ${ }^{1}$, P. Omoumi ${ }^{2}$, A. So ${ }^{1}$, N. Busso ${ }^{1} .{ }^{1}$ Lausanne University Hospital, CHUV, Department of Rheumatology, Lausanne, Switzerland; ' Lausanne University Hospital, CHUV, Radiology, Lausanne, Switzerland

Background: Fibrin(ogen) maintains inflammation in various disorders but has never been linked to cartilage damage in rheumatoid arthritis (RA) or other forms of inflammatory arthritis.

Objectives: To investigate the role of fibrin deposition on cartilage integrity in arthritis.

Methods: Fibrin deposition on knee cartilage was analyzed by immunohistochemistry in RA patients and in murine adjuvant-induced arthritis (AIA). In chondrocytes, fibrinogen expression $(F g \alpha, F g \beta, F g \gamma)$ and procoagulant activity were evaluated by qRT-PCR and turbidimetry respectively. Fibrin-induced catabolic genes were assessed by qRT-PCR in chondrocytes. Fibrin-mediated chondro-synovial adhesion (CSA) with subsequent cartilage tears was studied in co-cultures of human RA cartilage with autologous synoviocytes, in the AIA model, and by MRI. The link between fibrin and calcification was examined in human RA cartilage stained for calcific deposits and in vitro in fibrinogen-stimulated chondrocytes.

Results: Fibrin deposition on cartilage correlated with the severity of cartilage damage in human RA explants and in AIA wildtype (WT) mice, while fibrinogen deficient $\left(\mathrm{Fg}^{-/}\right)$mice were protected. Accordingly, fibrin upregulated catabolic enzymes (Adamts5 and Mmp13) in chondrocytes. Secondly, CSA was present in fibrin-rich and damaged cartilage in AIA WT but not in $\mathrm{Fg}^{-/-}$mice. In line, autologous human synoviocytes, cultured on RA cartilage explants, adhered exclusively to fibrin-positive degraded areas. Gadolinium-enhanced MRI of human joints showed contrast-enhancement along cartilage surface in RA patients but not in controls. Finally, fibrin co-localized with calcification in human RA cartilage and triggered chondrocyte mineralization inducing pro-calcification genes (Anx5, Pit1, Pc1) and cytokine (IL-6). Although at a much lesser extent, we observed similar fibrin-mediated mechanisms in osteoarthritis (OA)

Conclusion: Fibrin deposition directly impacts on cartilage integrity via induction of catabolism, mechanical stress, and calcification. Potentially, fibrin is a key factor of cartilage damage occurring in RA as a secondary consequence of inflammation.
Disclosure of Interests: None declared

DOI: 10.1136/annrheumdis-2021-eular.2521

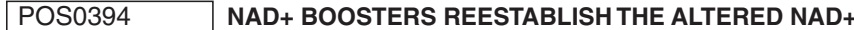 METABOLISM OF LEUKOCYTES FROM RHEUMATOID ARTHRITIS PATIENTS IMPROVING THEIR OXIDATIVE, APOPTOTIC AND INFLAMMATORY STATUS}

C. Perez-Sanchez ${ }^{1}$, L. M. Sánchez-Mendoza², M. D. C. Abalos-Aguilera', N. Barbarroja Puerto ${ }^{1}$, M. Luque-Tévar ${ }^{1}$, A. M. Patiño-Trives ${ }^{1}$, I. Arias de la Rosa', J. A. Moreno², M. I. Burón², J. A. González-Reyes², E. Collantes Estevez ${ }^{1}$, A. Escudero Contreras ${ }^{3}$, C. Lopez-Pedrera ${ }^{1}$, J. M. Villalba ${ }^{2}{ }^{1}{ }^{1} I M I B I C$, Rheumatology Service, IMIBIC/Reina Sofia Hospital/University of Cordoba, Cordoba, Spain, Córdoba, Spain; ${ }^{2}$ IMIBIC, Department of Cell Biology, Physiology and Immunology, University of Cordoba, Campus de Excelencia Internacional Agroalimentario, ceiA3, Córdoba, Spain, Córdoba, Spain; ${ }^{3}$ IMIBIC, Rheumatology Service, IMIBIC/Reina Sofia Hospital/University of Cordoba, Cordoba, Spain, Cordoba, Spain

Background: $\mathrm{NAD}^{+}$is an important cofactor and second messenger for multiple cellular processes that exhibits antioxidant, anti-apoptotic and anti-inflammatory properties. Pre-clinical studies in animal models of Rheumatoid Arthritis (RA) have demonstrated the therapeutic potential of $\mathrm{NAD}^{+}$boosters in the control of the disease activity. However, to date no studies have been set up to evaluate the $\mathrm{NAD}+$ metabolism and the therapeutic effects of NAD+ boosters in RA patients. Objectives: 1- To study the $\mathrm{NAD}^{+}$metabolism in RA patients and its association with key clinical features. 2- To evaluate the effect of anti-TNF therapy in the $\mathrm{NAD}+$ metabolism.

3- To analyze the beneficial effects of $\mathrm{NAD}^{+}$boosters in leukocytes from active RA patients.

Methods: Plasma and PBMCs were purified from 100 RA patients and 50 healthy donors (HDs). Moreover, an additional cohort of 50 RA patients treated with AntiTNF therapy was analyzed before and after 6 months of treatment. NAD ${ }^{+}$levels were determined by using the NAD/NADH-Glo Assay. $\mathrm{NAD}^{+}$-consuming genes expression were analyzed by RT-PCR. In parallel, PBMCs from eight HDs and eight active RA patients were treated ex vivo with $1 \mathrm{mM}$ of $\mathrm{NAD}^{+}$boosters including nicotinamide (NAM), nicotinamide riboside (NR), and nicotinamide mononucleotide (NMN). After 24 hours, intracellular reactive oxygen species (ROS) levels (DFCHDA) and the percentage of apoptotic PBMCs (annnexin V/PI) were assessed by flow cytometry. Lastly, a panel of key pro-inflammatory genes were evaluated by RT-PCR.

Results: $\mathrm{NAD}^{+}$and $\mathrm{NADH}$ levels were significantly reduced in plasma and PBMCs of RA patients compared with HDs and directly related to disease activity (DAS28, CDAI, SDAI). Accordingly, the expression levels of genes involved in the consumption of NAD ${ }^{+}$such as SIRT-1, CD38 and PARP-1 were found up-regulated in PBMCs from RA patients. Anti-TNF therapy for 6 months restored the altered NAD+ levels towards those showed by HDs. Furthermore, the clinical response promoted by Anti-TNF therapy (changes in DAS28) correlated with changes in NAD+ levels. The in vitro treatments of PBMCs isolated from active RA patients with $\mathrm{NAD}^{+}$boosters significantly increased the $\mathrm{NAD}^{+}$levels and promoted a deep reduction of intracellular ROS levels, the percentage of apoptotic cells and the expression levels of key inflammatory mediators, such as IL-6, IL-8, IL-1ß, TNF- $\alpha$, CCL2, IL-23, and STAT-3.

Conclusion: 1. $\mathrm{NAD}^{+}$metabolism is altered and associated with the disease activity of RA patients, involving both, reduced $\mathrm{NAD}^{+}$levels and increased expression of $\mathrm{NAD}^{+}$-consuming genes. 2. Anti-TNF therapy restored NAD+ levels, which were directly linked to the clinical effectiveness. 3. NAD ${ }^{+}$boosters reduced the oxidative, apoptotic and inflammatory profiles of RA leukocytes through the parallel increase of intracellular NAD ${ }^{+}$levels. Thus, NAD ${ }^{+}$boosters might be considered novel therapeutic tools for RA patients.

Acknowledgements: Supported by PI18/00837, RIER RD16/0012/0015 RTI2018-100695-B-I00, P18-RT-4264 and CVI276, co-funded with FEDER.

Disclosure of Interests: None declared

DOI: 10.1136/annrheumdis-2021-eular.2580

\section{POS0395 \\ ANTI-ACETYLATED PROTEIN ANTIBODIES IN RHEUMATOID ARTHRITIS (RA): CLUES FOR THE STARTING POINT OF AUTOANTIBODY RESPONSES IN RA}

T. J. van Wesemael ${ }^{1}$, A. L. Dorjée ${ }^{1}$, T. Huizinga $^{1}$, A. van der Helm - van Mil ${ }^{1}$, R. Toes ${ }^{1}$, D. van der Woude ${ }^{1}$. 'Leiden University Medical Center, Rheumatology, Leiden, Netherlands

Background: Rheumatoid arthritis (RA) is characterized by autoantibodies such as rheumatoid factor (RF) and anti-modified protein autoantibodies (AMPAs) like anti-citrullinated protein antibodies (ACPA) and anti-carbamylated protein 
antibodies (anti-CarP). Recently, another AMPA: anti-acetylated protein antibodies (AAPA) have been found in RA patients [1]. The prevalence of AAPA antibodies and their isotypes have yet to be determined. Since isotype profiles reflect the breadth of an immune response, the prevalence of AAPA isotypes in arthritis patients with and without RA can help to understand the relevance of this autoantibody response in RA.

Objectives: To describe the prevalence of AAPA isotypes in arthritis patients with and without RA.

Methods: In 650 RA patients fulfilling the 1987 RA criteria and 555 non-RA arthritis patients from the Leiden Early Arthritis Cohort, baseline serum samples were screened by ELISA for IgG, IgM and IgA to an acetylated- and control peptide that was based upon the CCP-2 backbone. The cutoff for positivity was based on 80 controls (mean $+2 \mathrm{SD}$ ). A sample was considered positive if it was above the cutoff and was 0.1 optical density higher on the acetylated peptide than on the control peptide.

Results: AAPA IgG was found in $36 \%$ of RA patients versus $6.7 \%$ of non-RA arthritis patients (figure 1a). Within RA patients, AAPA IgG antibodies were mostly present in the ACPA-(CCP-2) positive group (64\% in ACPA-positive, compared to $5 \%$ in ACPA-negative). Levels of AAPA IgG and IgA were higher in RA patients than in healthy controls and non-RA arthritis patients (figure 1b), however, surprisingly, no difference in levels was found for IgM.

When isotype profiles in AAPA- positive arthritis patients were compared, patients

Table 1. Anti-acetylated protein antibody (AAPA) isotype overlap in AAPA positive patients.

\begin{tabular}{|c|c|c|}
\hline AAPA isotype & RA patients $(=310) n(\%)$ & Non-RA arthritis patients $(n=106) n(\%)$ \\
\hline $\lg G+\lg M-\lg A-$ & 115 (37.1) & $28(5.1)$ \\
\hline $\lg G-\lg M+\lg A-$ & $52(16.8)$ & $48(8.7)$ \\
\hline IgG-IgM-IgA+ & $14(4.5)$ & $13(2.3)$ \\
\hline $\lg G+\lg M+\lg A-$ & $24(7.7)$ & $3(0.5)$ \\
\hline $\lg G+\lg M-\lg A+$ & 37 (11.9) & $4(0.7)$ \\
\hline $\lg G-\lg M+\lg A+$ & $9(2.9)$ & $8(1.4)$ \\
\hline $\lg G+\lg M+\lg A+$ & $59(19.0)$ & $2(0.4)$ \\
\hline
\end{tabular}

AAPA: anti-acetylated protein antibodies, RA: rheumatoid arthritis

with RA were more often positive for two or more isotypes then patients without RA, and thus displayed considerably more overlap in AAPA isotypes compared to non-RA patients (table 1). Intriguingly, IgM AAPA was the most prevalent isotype in non-RA patients, versus IgG in RA patients.

Conclusion: AAPA are detected in one third of RA patients, and mainly in the ACPA-positive subgroup. The predominance of IgM AAPA in non-RA arthritis patients and healthy controls suggests that healthy persons can develop AAPA IgM without the development of RA. These results also suggest that in healthy individuals, AAPA responses can occur, but do not mature past the IgM-stage, while in RA patients, the AAPA-response does mature and might form a "starting point" for development of other AMPA leading to the concurrent present of several AMPA in disease.

REFERENCES:

[1] Juarez, M., et al., Identification of novel antiacetylated vimentin antibodies in patients with early inflammatory arthritis. Ann Rheum Dis, 2016. 75(6): p. 1099-107.

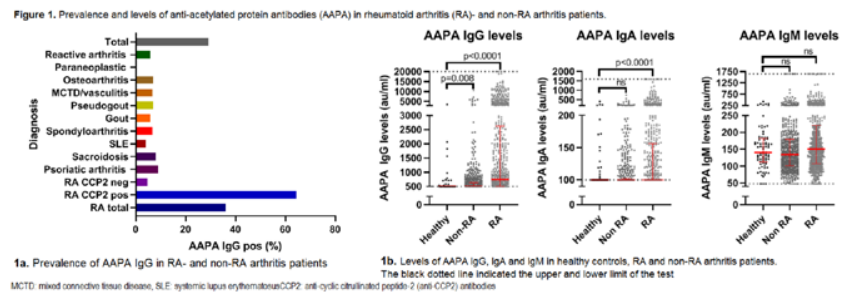

Disclosure of Interests: None declared

DOI: 10.1136/annrheumdis-2021-eular.2777

\section{POS0396 IS ASSOCIATED WITH THE CHANGES OF INTESTINAL MICROBIOTA IN PATIENTS WITH RHEUMATOID ARTHRITIS}

R. Wu ${ }^{1}$, J. An ${ }^{1}$, T. Ding ${ }^{1}$, H. Xue ${ }^{1}$, X. F. Li ${ }^{1}$, C. Wang ${ }^{1} .{ }^{1}$ The Second Hospital of Shanxi Medical University, The Department of Rheumatology, Taiyuan, China

Background: Rheumatoid arthritis (RA) is a systemic autoimmunity inflammation disease characterized with chronic aggressive arthritis and the presence of abnormal antibodies. Several observations showed that the breakdown of immune tolerance caused by many complex interactions was involved in the development of $\mathrm{RA}^{[1]}$. However, the pathogenesis of RA remained unclear. It has been confirmed that the imbalance of Th17 and Treg cells play a crucial role in destroying immune tolerance ${ }^{[2]}$. Besides, researches showed that intestinal microbiota can influence host immunity by acting on the immune cells to play pro-inflammatory or anti-inflammatory effect, and in turn immune system can also regulate the microbiota ${ }^{[3,4]}$. Thus, a frontier point of view in the field of rheumatism, immune microecology, was proposed, which is a novel concept for the breakdown of immune tolerance. Studies have confirmed that there was an imbalance of intestinal microbiota in patients with RA ${ }^{[4]}$. But the relationship between the $C D 4+T$ subsets cells and intestinal microbiota in RA is unknown.

Objectives: We detected and compared the absolute number of CD4+T cells subsets in the peripheral blood and the proportion or abundance of intestina microbiota in patients with RA and healthy adults, and then analyzed the relationship between them to explore the role of CD4+T cells subsets and intestinal microbiota in the pathogenesis of RA.

Methods: We collected the sample of stool and blood from 15 patients with RA hospitalized at the Second Hospital of Shanxi Medical University and 8 age and gender-matched healthy controls $(\mathrm{HC})$. The absolute number of $\mathrm{CD} 4+\mathrm{T}$ cells subsets including Th1, Th2, Th17 and Treg cells were detected by flow cytometry. The 16S rRNA in the stool specimens were sequenced by the Roche/45 high-throughput sequencing platform. We analyzed whether there was correlarion between CD4+T subsets cells and intestinal microbiota.

Results: Patients with RA had a higher level of Christensenellaceae and a lower level of Pseudomonadaceae as compared with those of $\mathrm{HCs}$ at the family level $(p<0.05)$. And at the genus level, the patients with RA had higher levels of Ruminococcus torques, Christensenellaceae R-7, Ruminiclostridium 9 and Ruminococcus 1 compared with those of $\mathrm{HCs}$ $(p<0.05)$ (Figure 1).And the Ruminococcus torques at the genus level was negative correlated with the absolute number of Treg cells $(p<0.001)$ (Figure 2).

Conclusion: The results here suggested that there were different proportion or abundance of intestinal microbiota between the patients with RA andHCs. And the changes of intestinal microbiota such as Ruminococcus torques were associated with Treg cells, further indicating that the imbalance of intestinal microbiota in RA can destory the immune tolerance. The above results uncovered that the intestinal microbiota had immunomodulatory function, which may be the upstream mechanism participated in the pathogenesis of RA.

\section{REFERENCES:}

[1] Weyand CM, Goronzy JJ. The immunology of rheumatoid arthritis. Nat Immunol 2021, 22(1): 10-18.

[2] Weyand CM, Goronzy JJ. Immunometabolism in the development of rheu matoid arthritis. Immunol Rev 2020, 294(1): 177-187.

[3] Brown EM, Kenny DJ, Xavier RJ. Gut Microbiota Regulation of T Cells During Inflammation and Autoimmunity. Annu Rev Immunol 2019, 37: 599-624.

[4] du Teil Espina M, Gabarrini G, Harmsen HJM, Westra J, van Winkelhoff AJ, van Dijl JM. Talk to your gut: the oral-gut microbiome axis and its immunomodulatory role in the etiology of rheumatoid arthritis. FEMS Microbiol Rev 2019, 43(1).
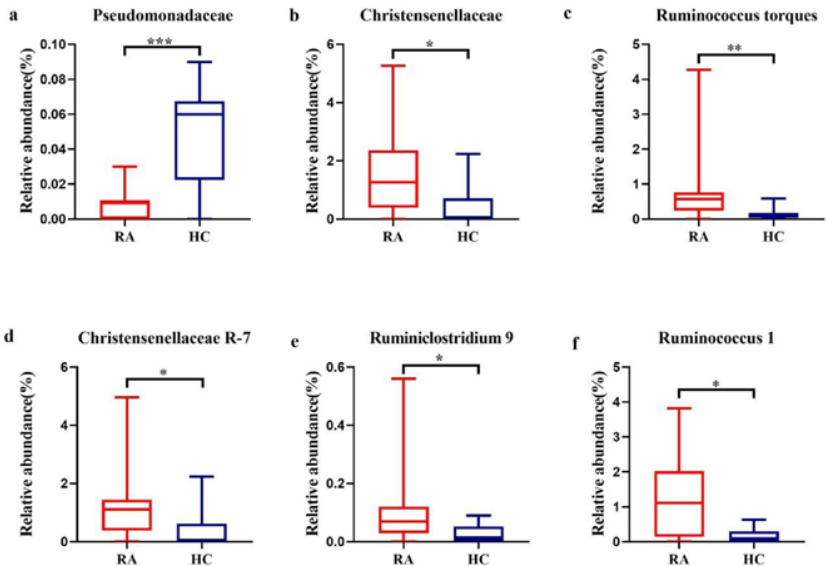

Figure 1. At the family level (a-b) and the genus level(c-f), the relative abundance of intestina microbiota in patients with RA and HCs were different. Data were expressed as median (Q1, Q3) and analyzed by Wilcoxon test. ( ${ }^{\star * *} P<0.001,{ }^{* *} P<0.01$ and $\left.{ }^{*} P<0.05\right)$. 